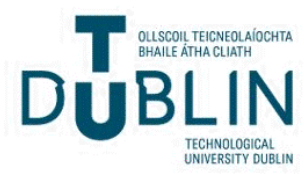

Technological University Dublin

ARROW@TU Dublin

Articles

Antenna \& High Frequency Research Centre

2013-4

Detecting Point-Like Sources of Unknown Frequency Spectra

\author{
Raffaele Solimene \\ Seconda Università di Napoli \\ Giuseppe Ruvio \\ Technological University Dublin \\ Angela Dell'Aversano \\ Seconda Università di Napoli
}

See next page for additional authors

Follow this and additional works at: https://arrow.tudublin.ie/ahfrcart

Part of the Diagnosis Commons, and the Systems and Communications Commons

\section{Recommended Citation}

Solimene, R. et al. (2013) Detecting point-like sources of unknown frequency spectra, Progress In Electromagnetics Research B, Vol. 50, 347-364, doi:10.2528/PIERB13030414

This Article is brought to you for free and open access by the Antenna \& High Frequency Research Centre at ARROW@TU Dublin. It has been accepted for inclusion in Articles by an authorized administrator of ARROW@TU

Dublin. For more information, please contact

arrow.admin@tudublin.ie, aisling.coyne@tudublin.ie, gerard.connolly@tudublin.ie.

Funder: SFI 


\section{Authors}

Raffaele Solimene, Giuseppe Ruvio, Angela Dell'Aversano, Antonio Cuccaro, Max Ammann, and Rocco Pierri 


\title{
DETECTING POINT-LIKE SOURCES OF UNKNOWN FREQUENCY SPECTRA
}

\author{
Raffaele Solimene ${ }^{2}$, Giuseppe Ruvio ${ }^{1,2, *}$, \\ Angela Dell'Aversano², Antonio Cuccaro' ${ }^{2}$, \\ Max J. Ammann ${ }^{3}$, and Rocco Pierri ${ }^{2}$ \\ ${ }^{1}$ Trinity College of Dublin, CTVR, Dunlop Oriel House, Dublin 2, \\ Ireland \\ ${ }^{2}$ Dipartimento di Ingegneria Industriale e dell'Informazione, Seconda \\ Università di Napoli, via Roma 29, Aversa I-81031, Italy \\ ${ }^{3}$ Antenna \& High Frequency Research Centre, Dublin Institute of \\ Technology, Kevin Street, Dublin 8, Ireland
}

\begin{abstract}
The problem of detecting point-like sources whose frequency spectrum is unknown is addressed. Limitations of singlefrequency approaches are identified by analytical as well as numerical arguments. To overcome these limits, different multifrequency approaches which combine frequency data incoherently are compared. In particular, a novel multifrequency MUSIC-like algorithm based on interferometric concepts is proposed. Results show that the algorithm outperforms other methods under comparison.
\end{abstract}

\section{INTRODUCTION}

Retrieving a current source from its radiated field is an ill-posed inverse problem [1]. This is due to the compactness of the radiation operator. Moreover, such an operator is also non-injective [2] apart for cases where the golden rule is not violated [3], as for example for strip sources [4], or when additional constraints are imposed on the solution [5]. Accordingly, the resolving kernel is not a Dirac delta function and this implies that the inversion procedure fails to reproduce the original current distribution: indeed only a smoothed version of the generalized solution can be retrieved [1]. For point-like sources, which are of concern in this analysis, this leads to finite spatial resolution while attempting to localize them.

Received 4 March 2013, Accepted 11 April 2013, Scheduled 14 April 2013

* Corresponding author: Giuseppe Ruvio (ruviog@tcd.ie). 
As distinct from inverse scattering problems, in an inverse source scenario it is not formally possible to use some diversities to collect more independent data [6]. This would be the case of multiple scene illuminations (i.e., primary sources located at different positions) as the source locations are just the unknowns. Moreover, frequency diversity can be employed to restore uniqueness only if the source frequency behaviour is a priori known. Unfortunately, the achievable resolution is still limited due to the ill-posedness which imposes a tradeoff between resolution and stability against uncertainties [6]. Moreover, in the case of a lossy and/or dispersive background medium some filtering is also generally required to compensate for further loss of resolution and to restore image dynamic range [7].

In this paper, the detection and localization of point-like sources whose frequency behavior is unknown is considered. In particular, the role of number of measurement points, imprecise knowledge of host medium dielectric properties and the effect of losses are investigated.

First, limitations of the single-frequency approach are highlighted. To overcome these problems a multi-frequency configuration can be addressed [8]. In particular, the multi-frequency MUSIC-like detection algorithm proposed in [9] is adopted and compared to other existing methods. It is shown that the new method exhibits better performance.

The plan of the paper is the following. Section 2 is dedicated to showing achievable performance and drawbacks affecting single frequency detection algorithms. This allows gathering useful information to devise the multi-frequency detection scheme presented in Section 3. Three appendices support mathematical arguments employed throughout the paper. Finally, conclusions end the article.

\section{SINGLE-FREQUENCY CASE}

In order to perform point-like source localization, the first step consists of establishing the mathematical model between the sources' positions and the radiated field that must be inverted. This can be expressed in terms of the radiation integral which for a two-dimensional scalar case writes as

$$
E\left(\underline{r}_{O}, \omega\right)=\int_{D} G\left(\underline{r}_{O}, \underline{r}, \omega\right) \gamma(\underline{r}, \omega) d \underline{r}
$$

where $E\left(\underline{r}_{O}, \omega\right)$ and $\gamma(\underline{r}, \omega)$ are the radiated field and the current distribution, respectively. $D$ is the spatial domain where the sources are assumed to reside, $\underline{r}_{O}$ the observation point, $\omega$ the angular frequency, and $G\left(\underline{r}_{O}, \underline{r}, \omega\right)$ the background Green's function. In 
particular,

$$
\gamma(\underline{r}, \omega)=\sum_{n=1}^{N_{S}} I_{n}(\omega) \delta\left(\underline{r}-\underline{r}_{n}\right)
$$

where $N_{S}$ is the number of point-like sources, and $\underline{r}_{n}$ and $I_{n}(\omega)$ are their positions and frequency spectra.

As both $E\left(\underline{r}_{O}, \omega\right)$ and $\gamma(\underline{r}, \omega)$ depend on frequency, all the frequencies cannot be simultaneously exploited for source reconstruction as increasing data would also entail increasing the number of unknowns.

The simplest way to proceed is to exploit single-frequency data. This allows to disregard dispersion, losses and source frequency behaviour. By contrast, this also entails an intrinsic limit on the achievable performance as the operator to be inverted is not injective. In this section, these limitations are studied.

Consider the radiated field be collected over $N_{O}$ points (with $N_{O}>N_{S}$ ) at a fixed frequency $\omega$, the field vector can be written as

$$
\mathbf{E}(\omega)=\mathbf{A}(\omega) \mathbf{b}(\omega)
$$

where $\mathbf{E}(\omega)=\left[E\left(\underline{r}_{O 1}, \omega\right) E\left(\underline{r}_{O 2}, \omega\right), \ldots, E\left(\underline{r}_{O N_{O}}, \omega\right)\right]^{T}, \mathbf{b}(\omega)=$ $\left[I_{1}(\omega), I_{2}(\omega), \ldots, I_{N_{S}}(\omega)\right]^{T}$ (note that the vector $\mathbf{b}(\omega)$ is unknown), with $(\cdot)^{T}$ denoting the transpose, and $\mathbf{A}(\omega)$ is the $N_{O} \times N_{S}$ matrix propagator (indeed a discrete version of Equation (1)) whose $n$-th column has the form

$$
\mathbf{A}^{n}\left(\underline{r}_{n}, \omega\right)=\left[G\left(\underline{r}_{O 1}, \underline{r}_{n}, \omega\right), G\left(\underline{r}_{O 2}, \underline{r}_{n}, \omega\right), \ldots, G\left(\underline{r}_{O N}, \underline{r}_{n}, \omega\right)\right]^{T}
$$

The corresponding correlation matrix is built up as follows

$$
\mathbf{R}(\omega)=\mathbf{E}(\omega) \mathbf{E}^{H}(\omega)=\mathbf{A}(\omega) \mathbf{B}(\omega) \mathbf{A}^{H}(\omega)
$$

where $\mathbf{b}^{H}(\omega)$ and $\mathbf{A}^{H}(\omega)$ are the Hermitian vector and matrix of $\mathbf{b}(\omega)$ and $\mathbf{A}(\omega)$, respectively, and $\mathbf{B}(\omega)=\mathbf{b}(\omega) \mathbf{b}^{H}(\omega)$.

According to [10], sources can be localized by finding the steering vectors which are orthogonal to the so called noise subspace. This requires computing the eigenspectrum of $\mathbf{R}(\omega)$ and the steering vectors, which in turn consist of columns $\mathbf{A}_{k}(\omega)=$ $\mathbf{A}_{k}\left(\underline{r}_{k}, \omega\right) /\left\|\mathbf{A}_{k}\left(\underline{r}_{k}, \omega\right)\right\|$ being evaluated in correspondence of the trial position $\underline{r}_{k}$ within the spatial domain $D$. Hence, sources' positions are identified where the pseudospectrum

$$
\phi\left(\underline{r}_{k} ; \omega\right)=1 /\left\|P\left[\mathbf{A}_{k}(\omega)\right]\right\|^{2}
$$

peaks, with $P[\cdot]$ being the projection operator onto the noise subspace.

A crucial point concerning Equation (6) is that the unknown sources are deterministic. Therefore $\mathbf{R}(\omega)$ is rank-deficient (regardless 
of the employed frequency) with rank equal to one (without considering the noise of course). Rank deficiency of $\mathbf{R}(\omega)$ entails performance degradation. Some methods to restore the rank of the correlation matrix in conjunction with multi-frequency illumination [11] or a multiview configuration [12] can be employed in inverse scattering. However, these methods here cannot be followed as frequency source behaviour is unknown and multi-view configuration is not allowed.

The achievable performance can be equivalently and more conveniently studied by employing the projector over the signal subspace, $Q(\cdot)$, instead of $P(\cdot)$, that is

$$
\left\|P\left[\mathbf{A}_{k}(\omega)\right]\right\|^{2}=\left\|(I-Q)\left[\mathbf{A}_{k}(\omega)\right]\right\|^{2}
$$

for only the significant singular vector is necessary in $Q(\cdot)$ and hence to implement the detection algorithm.

Let us name $\mathbf{u}_{1}(\omega)$ the singular vector corresponding to the theoretically only singular value which is different from zero. As the columns of the propagator $\mathbf{A}(\omega)$ span the range of $\mathbf{R}(\omega)$, it results that

$$
\mathbf{u}_{1}(\omega)=\sum_{n=1}^{N_{S}} \alpha_{n}(\omega) \mathbf{A}^{n}\left(\underline{r}_{n} ; \omega\right)
$$

which can then be rearranged as

$$
\mathbf{u}_{1}(\omega)=\mathbf{E}(\omega) /\|\mathbf{E}(\omega)\|
$$

From Equations (8) and (9), it is seen that the eigenspectrum computation of $\mathbf{R}(\cdot)$ is indeed not necessary since

$$
\left\|(I-Q)\left[\mathbf{A}_{k}(\omega)\right]\right\|^{2}=1-\left|\left\langle\mathbf{A}_{k}(\omega), \mathbf{u}_{1}(\omega)\right\rangle\right|^{2}
$$

where $\langle\cdot, \cdot\rangle$ denotes the Hermitian scalar product in the data space. Now, it is recognized that in order to understand how detection algorithm behaves, it is sufficient to study the term $\left|\left\langle\mathbf{A}_{k}(\omega), \mathbf{u}_{1}(\omega)\right\rangle\right|^{2}$.

\subsection{Single Source}

We start by considering a single scatterer embedded within a homogeneous medium with dielectric permittivity denoted as $\varepsilon_{B G}$. The radiated field is assumed being collected over a measurement curve $\Sigma$ consisting of a circle of radius $r_{O}$ that surrounds the investigation domain $D$. Being $\underline{r}_{S}$ the source position, following Equation (10), the projection $\cos \eta_{k S}$ of the steering vector $\mathbf{A}_{k}(\omega)$ corresponding to the position $\underline{r}_{k} \in D$ over $\mathbf{u}_{1}(\omega)$ writes as

$$
\begin{aligned}
\left\langle\mathbf{A}_{k}(\omega), \mathbf{u}_{1}(\omega)\right\rangle & =\cos \eta_{k S} \\
& =\frac{\left\langle H_{0}^{(2)}\left(k_{B G}\left|\underline{r}_{O}-\underline{r}_{k}\right|\right), H_{0}^{(2)}\left(k_{B G}\left|\underline{r}_{O}-\underline{r}_{S}\right|\right)\right\rangle}{\left\|H_{0}^{(2)}\left(k_{B G}\left|\underline{r}_{O}-\underline{r}_{k}\right|\right)\right\|\left\|H_{0}^{(2)}\left(k_{B G}\left|\underline{r}_{O}-\underline{r}_{s}\right|\right)\right\|}
\end{aligned}
$$


where $k_{B G}$ is the background wavenumber, and $H_{0}^{(2)}(\cdot)$ is the Hankel function of zero order and second kind, that is the relevant Green's function. For $\underline{r}_{k}$ and $\underline{r}_{S}$ not close to $\Sigma$, it can be shown (see Appendix A) that

$$
\cos \eta_{k S} \simeq J_{O}\left(k_{B G}\left|\underline{r}_{k}-\underline{r}_{S}\right|\right)
$$

Accordingly,

$$
\phi\left(\underline{r}_{k}, \omega\right)=1 /\left[1-J_{O}\left(k_{B G}\left|\underline{r}_{k}-\underline{r}_{S}\right|\right)^{2}\right]
$$

Equation (13) is important as it allows to point out that $\phi(\cdot)$ actually peaks on the source's location. Moreover, it shows how the MUSIClike method performs. Indeed, $\left|\left\langle\mathbf{A}_{k}(\omega), \mathbf{u}_{1}(\omega)\right\rangle\right|^{2}$ can be thought as a sort of weighted single-frequency migration because $\mathbf{A}_{k}(\omega)$ and $\mathbf{u}_{1}(\omega)$ are normalized vectors. Therefore, the result in Equation (12) is to be expected as it is consistent with diffraction limits. Instead, the pseudospectrum in Equation (13) enables finer localization basically because the dynamics of the reconstruction is very much enlarged.

In order to verify the theory so far discussed, an infinitely long cylindrical investigation domain is considered. Its radius is $D=50 \mathrm{~mm}$ and it is immersed into a background medium with $\varepsilon_{B G}=10 \varepsilon_{0}, \varepsilon_{0}$ being the dielectric permittivity of the free-space. The radiated field is collected over 18 positions taken uniformly over a concentric (with $D$ ) circle of radius equal to $55 \mathrm{~mm}$. The scenario is homogeneous with the permittivity of the cylindrical domain being the same as the background medium. The source to be detected is located at $\underline{r}_{S} \equiv$ $(0 ; 10) \mathrm{mm}$ with respect to the origin of the cylindrical geometry and the operating frequency is $2 \mathrm{GHz}$. In Figure 1 the results returned by Equations (12) and (13) are compared to those obtained by exploiting synthetic data provided by the FDTD based tool GPRMAX [13]. As can be seen, actual projection and source localization are very well approximated (reconstructions are given in $\mathrm{dB}$ scale). If a $-3 \mathrm{~dB}$ threshold is assumed to define resolution (as usually done) this would lead to an extremely fine resolution. In any case, the clutter pedestal is very low. This is just what is normally expected by a MUSIC-like algorithm.

\subsection{Unknown Background Medium}

Previous results have been derived under some previously mentioned ideal conditions. It is interesting to analyze how things are altered when such hypotheses are removed. Here, we start by considering the case where the background medium (still homogeneous) is not a priori known. This entails that the assumed $k_{B G}$ is different from the actual 


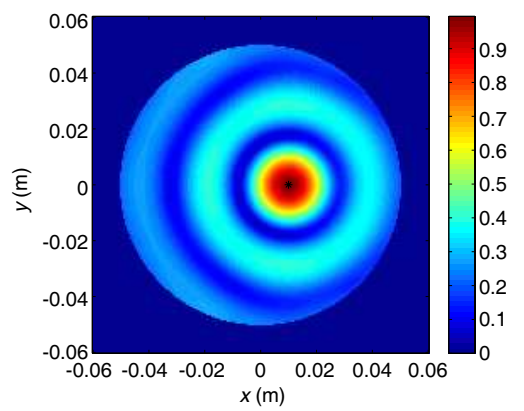

(a)

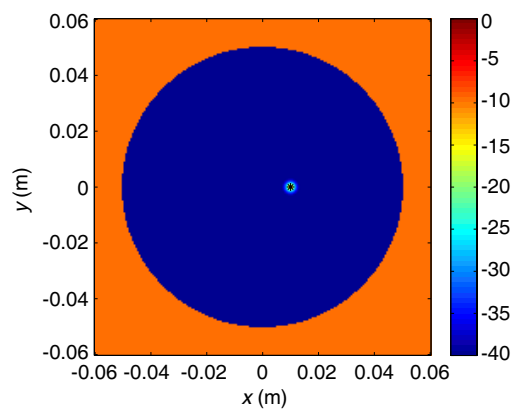

(c)

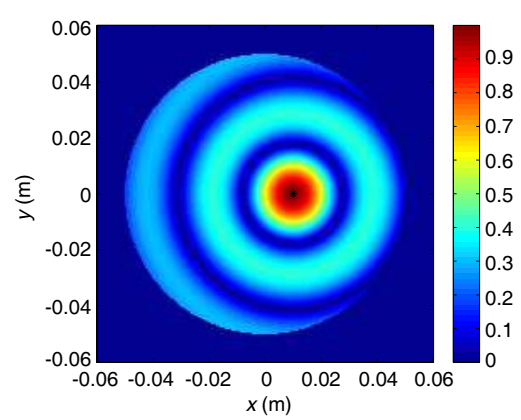

(b)

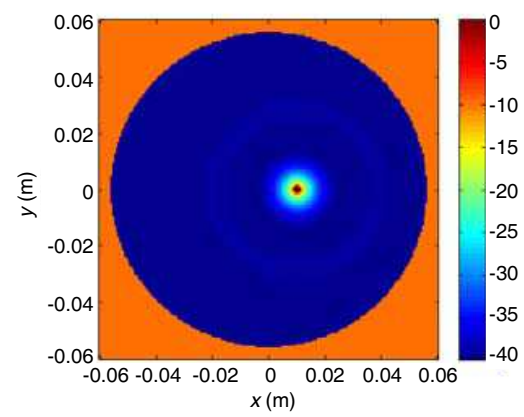

(d)

Figure 1. Assessing theoretical results of Equations (12) and (13). (a) Outcome of Equation (12). (b) Actual projection. (c) Outcome of Equation (13). (d) Actual pseudospectrum. In all the figures actual source's position is displayed as black asterisk. Figures 1(b) and $1(\mathrm{~d})$ were obtained by employing synthetic data at $2 \mathrm{GHz}$ and 18 measurement points.

one, denoted as $\tilde{k}_{B G}$. The corresponding steering vector projection is given by

$$
\cos \eta_{k S}=\frac{\left\langle H_{0}^{(2)}\left(k_{B G}\left|\underline{r}_{O}-\underline{r}_{k}\right|\right), H_{0}^{(2)}\left(k_{B G}\left|\underline{r}_{O}-\underline{r}_{S}\right|\right)\right\rangle}{\left\|H_{0}^{(2)}\left(k_{B G}\left|\underline{r}_{O}-\underline{r}_{k}\right|\right)\right\|\left\|H_{0}^{(2)}\left(\tilde{k}_{B G}\left|\underline{r}_{O}-\underline{r}_{s}\right|\right)\right\|}
$$

which can be expressed as (see Appendix B)

$$
\cos \eta_{k S}=J_{O}\left(k_{B G}\left|\underline{r}_{k}-\frac{\tilde{k}_{B G}}{k_{B G}} \underline{r}_{S}\right|\right)
$$

Equation (15) highlights the expected smearing and delocalization due to the mismatch between the assumed and the actual host medium. In 
particular, it is interesting to note that delocalization only occurs along the radial coordinate. This is clearly shown in Figure 2, where the same case as in Figure 1 is considered except for $\varepsilon_{B G}=2.5 \varepsilon_{0}$. According to Equation (15), the pseudospectrum must peak at $(0 ; 20) \mathrm{mm}$ instead of $\underline{r}_{S} \equiv(0 ; 10) \mathrm{mm}$ as it actually occurs.

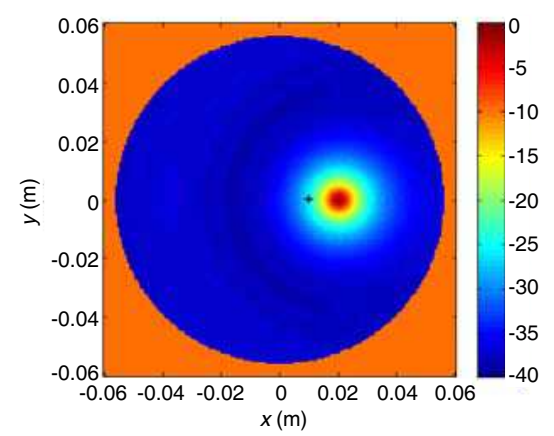

Figure 2. Role of unknown host medium. Actual source's position is marked with a black asterisk.

The host medium can be lossy. Hence it is worth investigating what happens when losses are not accounted for in the model. To this end, previous examples in Figure 1 are recalculated by considering conductivity losses with $\sigma_{B G}=0.75 \mathrm{~S} / \mathrm{m}$. This is a relatively high value which is specifically used to make the effect of losses more evident. The corresponding results are reported in Figure 3 where pseudospectra obtained by considering losses and without considering losses are shown. As can be seen, when losses are taken into account, the algorithm performs very much like the lossless case. However, when they are not considered, a certain loss of resolution and delocalization is observed. Indeed, loss of resolution is not so dramatic if the usual $-3 \mathrm{~dB}$ threshold is considered and delocalization becomes significant only for very high values of conductivity. However, the most important drawback is the reduction in the dynamic range for the pedestal clutter being almost doubled with respect to previous cases.

\subsection{Undersampled Data}

In practical situations the number of data samples is finite and in some cases it could be very low. In inverse scattering, for Time-ReversalMUSIC this seems not to be a problem provided that the number of data is greater than the scatterers. However, here an inverse source problem is under consideration. As mentioned above, this entails one 


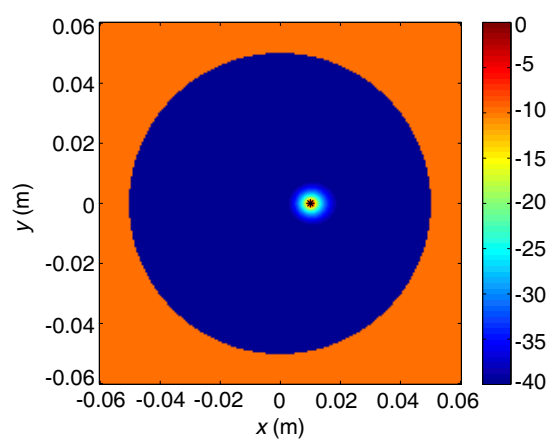

(a)

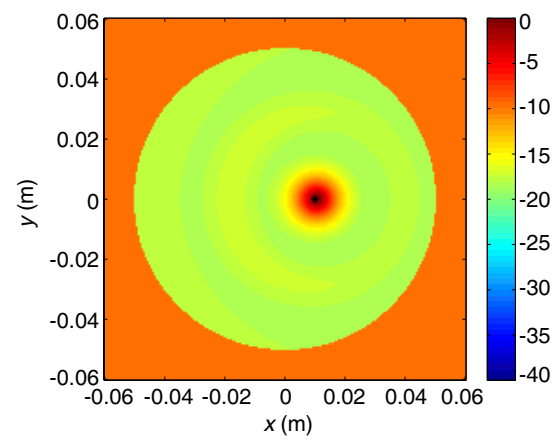

(b)

Figure 3. Role of conductivity loss. (a) Losses are accounted for. (b) Losses are not accounted for. Actual source's position is displayed as black asterisk.

single singular vector being involved. It is thus necessary to understand how the method works for limited data samples. Under the same assumptions and configuration as in Section 2.1, it can be shown that (see Appendix C)

$$
\begin{aligned}
\cos \eta_{k S} \cong & J_{O}\left(k_{B G}\left|\underline{r}_{S}-\underline{r}_{k}\right|\right)+\sum_{m \neq 0} j^{m N_{O}} J_{m N_{O}}\left(k_{B G}\left|\underline{r}_{S}-\underline{r}_{k}\right|\right) \\
& \times \exp \left[-j m N_{O} \arg \left(\underline{r}_{S}-\underline{r}_{k}\right)\right]
\end{aligned}
$$

with $N_{O}$ being the number of measurements.

From Equation (16) it is foreseen that the projection can be high not only for the steering vector corresponding to the source's actual position $\underline{r}_{S}$ but also elsewhere. That is where the terms of the series appearing on the right hand side interfere constructively. Hence, it is expected that spurious peaks will populate the reconstruction. Moreover, this number increases as $N_{O}$ decreases and/or frequency increases. This conclusion is clearly supported by the example shown in Figure 4 where in order to exacerbate replica occurrences only three measurement points were considered.

\subsection{Multiple Sources}

When more than one source populates the scene, the performance can undergoes severe degradation due to the rank deficiency of $\mathbf{R}(\omega)$. Indeed, under this circumstance the term $\left\|\left(I-Q_{i}\right)\left[\mathbf{A}_{k}(\omega)\right]\right\|^{2}$ is no longer null in correspondence of the sources' locations. This is because the singular vector $\mathbf{u}_{1}(\omega)$ is a linear combination of the sources' 


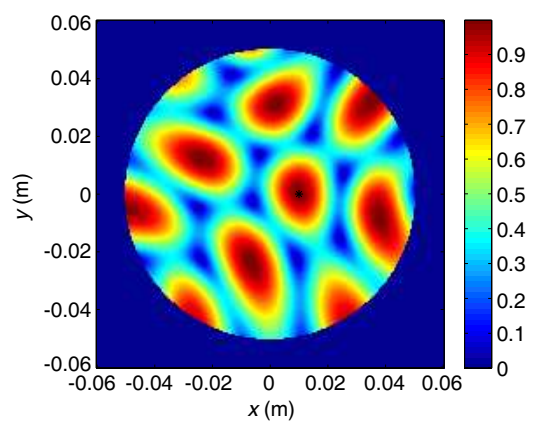

(a)

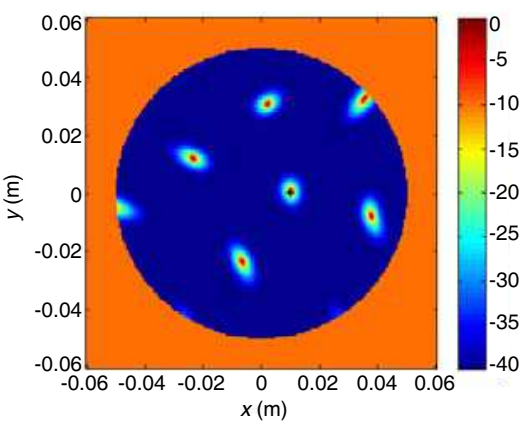

(b)

Figure 4. Undersampled data, $N_{O}=3$. (a) Projection over signal singular vector. (b) Corresponding pseudospectrum. Actual source's position is displayed as black asterisk.

steering vectors (see Equation (9)), hence none of them can be parallel to it. Moreover, these projections will assume generally different values unless their steering vectors lie on a cone whose axis coincides with $\mathbf{u}_{1}\left(\omega_{i}\right)$. The latter circumstance may depend on the sources' positions, losses within the background medium and aperture limited configuration. Therefore, rank deficiency can be responsible for a loss of resolution as well as of a dynamic range reduction.

More precisely, by considering the sources located at $\underline{r}_{n}$, with $n=1, \ldots, N_{S}$ and naming $\mathbf{A}_{n}(\omega)$ the corresponding normalized steering vectors, the projection of the steering vector corresponding to the $m$-th source is then given by

$$
=\frac{\sum_{n=1}^{N_{S}} I_{n}^{*}\left\langle H_{0}^{(2)}\left(k_{B G}\left|\underline{\mathbf{r}}_{O}-\underline{r}_{m}\right|\right), H_{0}^{(2)}\left(k_{B G}\left|\underline{r}_{O}-\underline{r}_{n}\right|\right)\right\rangle}{\sqrt{\left\|H_{0}^{(2)}\left(k_{B G}\left|\underline{r}_{O}-\underline{r}_{m}\right|\right)\right\|^{2}\left(\sum_{n=1}^{N_{S}}\left|I_{n}\right|^{2}\left\|H_{0}^{(2)}\left(k_{B G}\left|\underline{r}_{O}-\underline{r}_{n}\right|\right)\right\|^{2}\right.}}
$$

If the sources are well-resolved (which according to Equation (12) means that the sources are separated by more than the main lobe width of the Bessel function of zero order) then Equation (17) can be 
approximated as

$$
\cong \frac{\left\langle\mathbf{A}_{m}(\omega), \mathbf{u}_{1}(\omega)\right\rangle}{\sqrt{\left\|H_{0}^{(2)}\left(k_{B G}\left|\underline{r}_{O}-\underline{r}_{m}\right|\right)\right\|^{2} \sum_{n=1}^{N_{S}}\left|I_{n}\right|^{2}\left\|H_{0}^{(2)}\left(k_{B G}\left|\underline{r}_{O}-\underline{r}_{n}\right|\right)\right\|^{2}}}(18)
$$

The corresponding peseudospectrum is then given by

$$
\phi\left(\underline{r}_{m}, \omega\right)=\sum_{n=1}^{N_{S}} \alpha_{n m}\left|I_{n}\right|^{2} /\left[\sum_{n=1}^{N_{S}} \alpha_{n m}\left|I_{n}\right|^{2}-\left|I_{m}\right|^{2}\right]
$$

where $\alpha_{n m}=\left\|H_{0}^{(2)}\left(k_{B G}\left|\underline{r}_{O}-\underline{r}_{n}\right|\right)\right\|^{2} /\left\|H_{0}^{(2)}\left(k_{B G}\left|\underline{r}_{O}-\underline{r}_{m}\right|\right)\right\|^{2}$.

As can be seen, in the case of more than one scatterer the pseudospectrum no longer diverges at the scatterers' positions. Therefore, as anticipated above, even though the sources are wellresolved, a certain loss of dynamic range and resolution is expected. Accordingly, it is also expected that some sources are missed while reconstructing the scene.

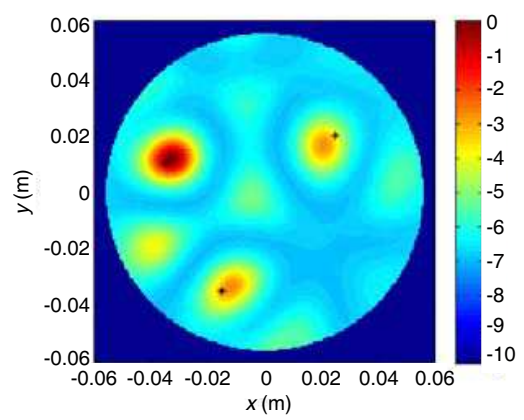

(a)

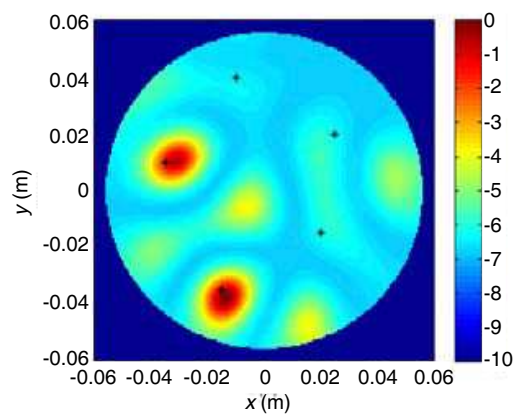

(c)

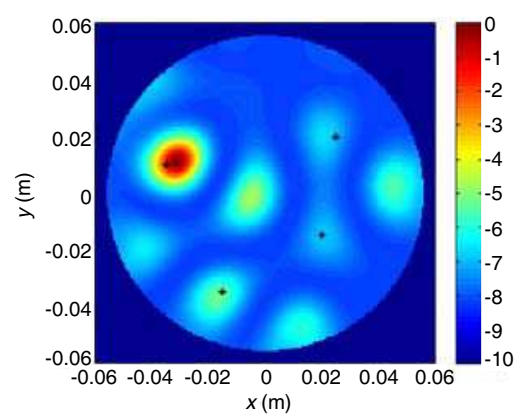

(b)

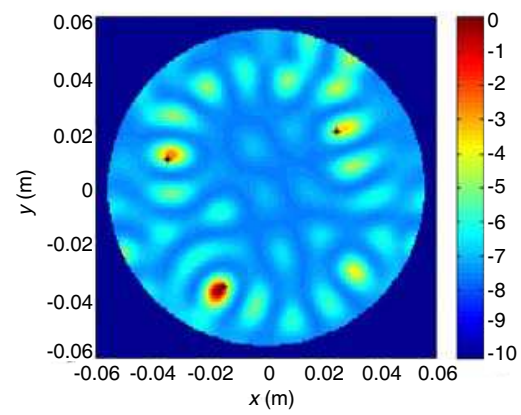

(d) 


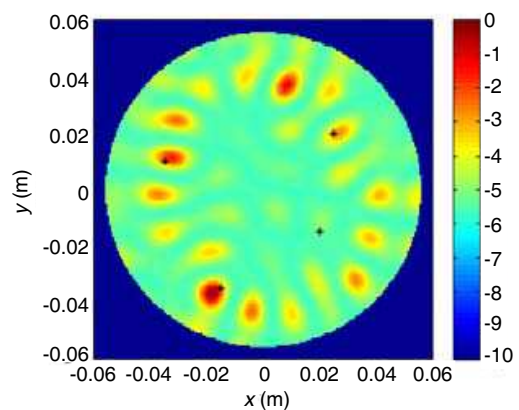

(e)

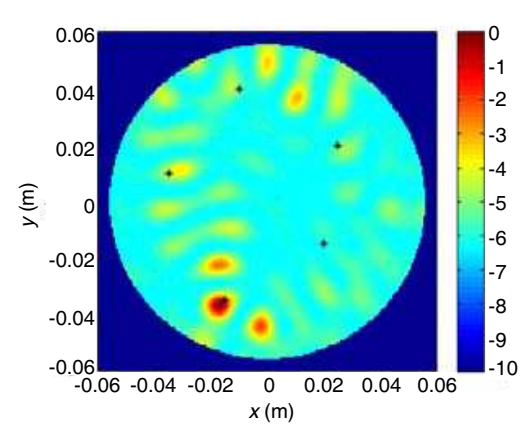

(f)

Figure 5. The case of multiple sources for the same configuration as in Figure 1. Pseudospectra on Figures 5(a), 5(b) and 5(c) are obtained with $f=2 \mathrm{GHz}$, whereas those on Figure 5(d), 5(e) and 5(f) with $f=4 \mathrm{GHz}$. Figures $5(\mathrm{a})$ and $5(\mathrm{~d})$ refer to three sources, Figures $5(\mathrm{~b})$ and $5(\mathrm{~d})$ to four sources and Figures $5(\mathrm{c})$ and $5(\mathrm{f})$ to five sources. Actual sources' positions are displayed as black asterisks.

The examples reported in Figure 5 confirm this discussion. These show the case of three, four and five sources under consideration. As can be seen, for the case of three sources all the sources are detected. However, as expected, pseudospectrum peaks are smeared and of different amplitude. The performance gets worse when sources' number increases. Indeed, apart from previously seen drawbacks, some sources are in this case completely missed and overwhelmed by spurious peaks. Finally, it is worth noticing that by increasing the working frequency, the performance does not improve.

\section{MULTIPLE-FREQUENCY CASE}

As shown above, a single-frequency approach suffers from two main limitations. First, when data are undersampled, many source's replica corrupt the pseudospectrum and, consequently, actual sources result indiscernible. Second, due to the rank deficiency of $\mathbf{R}(\omega)$, when multiple sources populate the scene, achievable performance quickly degrades.

Data collected at different frequencies can be employed to improve the performance of the single-frequency case. However, as the $I_{n}(\omega)$ are unknown, frequency information cannot be coherently combined. Alternatively, an incoherent approach can be followed. A simple way to achieve that is suggest in [9] and [14]: first pseudospectra at each 
single frequency are obtained, then, they are combined incoherently. In particular, following [14], the so-called wide-band MUSIC (WBMUSIC) is obtained as

$$
\Phi_{\text {sum }}\left(\underline{r}_{k}\right)=1 / \sum_{i=1}^{N_{f}}\left\|P_{i}\left[\mathbf{A}_{k}\left(\omega_{i}\right)\right]\right\|^{2}
$$

where $N_{f}$ is the number of adopted frequencies and $\omega_{i}$ is the $i$-th one.

Here, it is also explored a different strategy based on interferometric arguments [9]. In detail, the overall detection is built

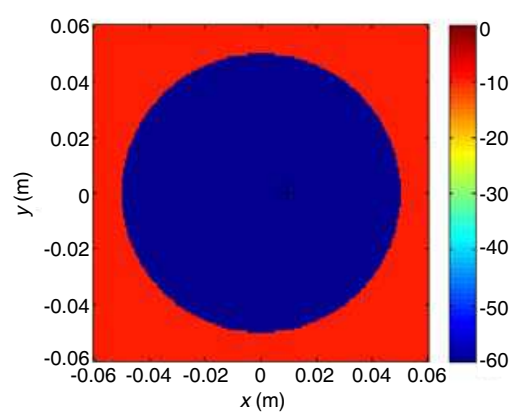

(a)

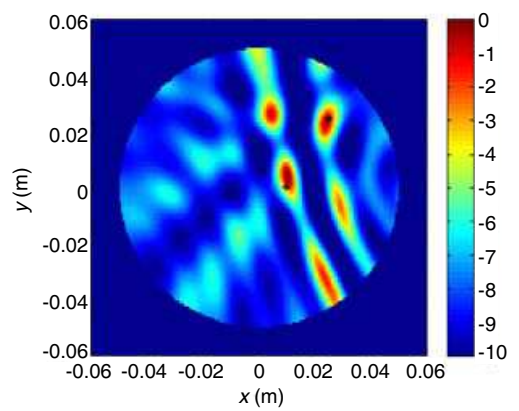

(c)

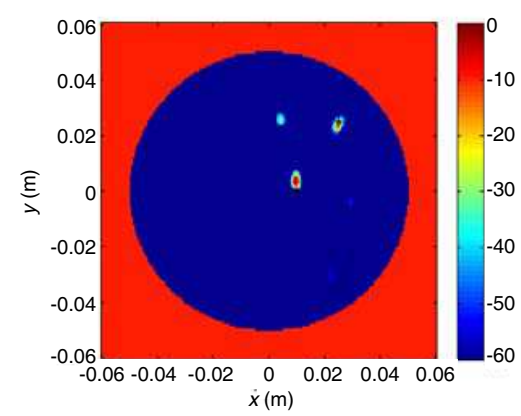

(b)

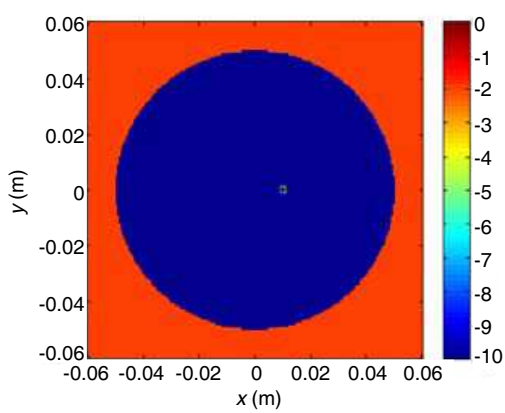

(d)

Figure 6. The case of undersampled data as in Figure 4 for multi-frequency data taken at 20 frequencies within the band 2$4 \mathrm{GHz}$. Figures 6(a) and 6(b) show I-MUSIC pseudospectra, whereas Figures 6(c) and 6(d) show those obtained by WB-MUSIC. Figures 6(a) and 6(c) refer to a single source, whereas Figures 6(b) and 6(d) to two sources. Actual sources' positions are displayed as black asterisks. 
up as

$$
\Phi_{p r d}\left(\underline{r}_{k}\right)=1 / \prod_{i=1}^{N_{f}}\left\|P_{i}\left[\mathbf{A}_{k}\left(\omega_{i}\right)\right]\right\|^{2}
$$

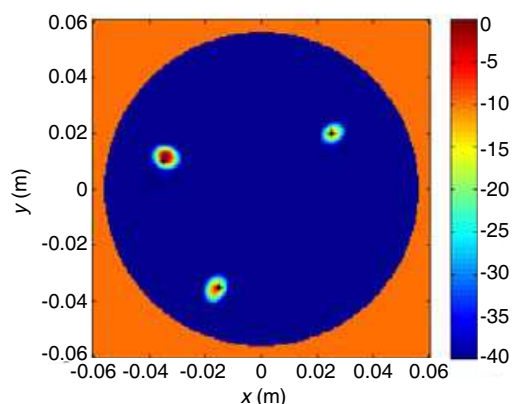

(a)

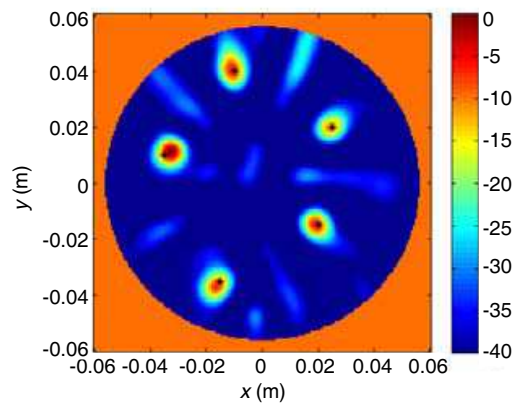

(c)

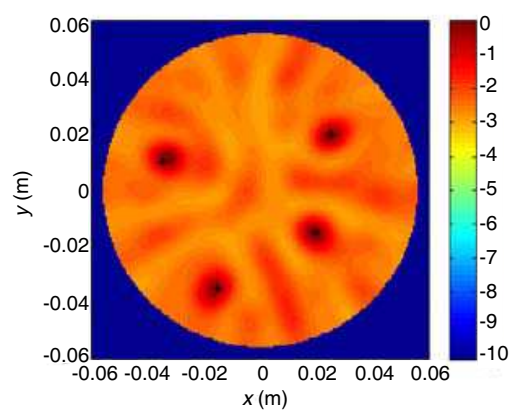

(e)

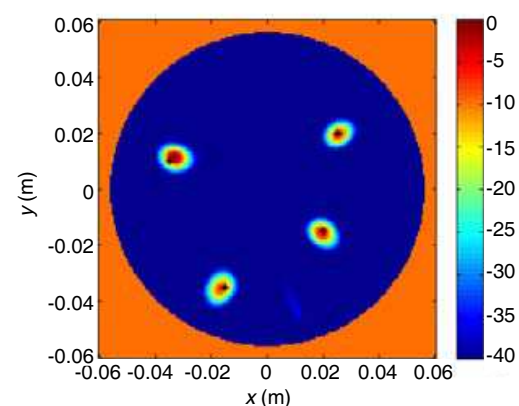

(b)

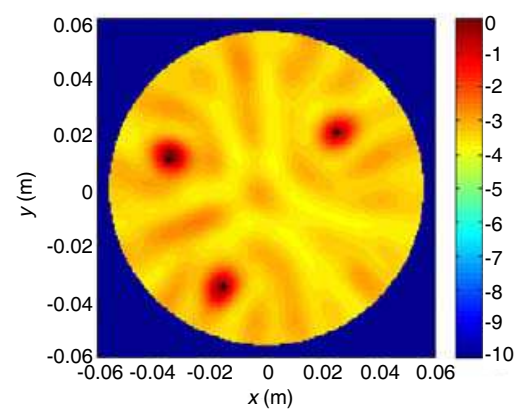

(d)

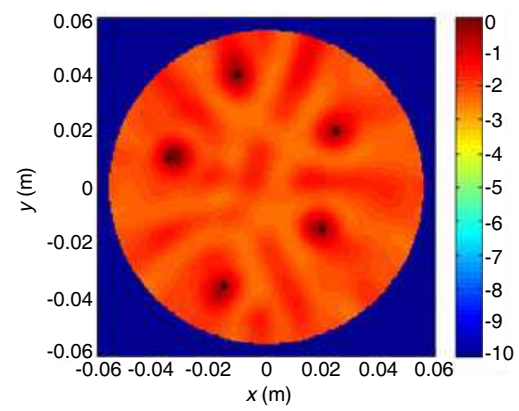

(f)

Figure 7. Reconstruction of multiple sources by a multifrequency configuration consisting of 20 frequency taken uniformly in the band $2-4 \mathrm{GHz}$. The number of measurement points and the background medium are the same as in Figure 1. Pseudospectra on Figures 7(a), 7 (b) and 7(c) are obtained by I-MUSIC, whereas those on Figures 7(d), $7(\mathrm{e})$ and $7(\mathrm{f})$ are obtained by WB-MUSIC. Actual sources' positions are displayed as black asterisks. 
Hence, it consists in multiplying pixel by pixel the pseudospectra obtained at different frequencies. In this paper this method is addressed as interferometric-MUSIC (I-MUSIC).

In order to check the effectiveness of (20) and (21) in overcoming single-frequency drawbacks, the cases of Figures 4 and 5 are rerun. However, now the radiated field is collected at twenty frequencies $\left(N_{f}=20\right)$ uniformly taken within the band $[2,4] \mathrm{GHz}$. Moreover, data were corrupted by an additive zero mean white Gaussian noise corresponding to the signal-to-noise ratio $\mathrm{SNR}=20 \log \|\mathbf{E}\|_{F} /\|N\|_{F}=$ $10 \mathrm{~dB}$, where $N$ is the noise and $\|\cdot\|$ the Freobenius norm over positions and frequencies.

In Figure 6 the undersampled case of Figure 4 is addressed for one and two sources, respectively. As can be seen, for the single source cases, both the multi-frequency methods allow a strong mitigation of replica occurrence. This is because, according to Equation (16), such artefact positions are frequency dependent and are hence averaged out when employing multi-frequency data. However, for the two sources scenario, I-MUSIC pseudospectrum is clearer.

The multiple source cases are addressed in Figure 7. Here, it can be clearly seen that all the sources are well detected and localized. Moreover, I-MUSIC proves to be superior to WB-MUSIC as the image dynamic ranges returned by the former method is much greater.

\section{CONCLUSIONS}

In this paper, the detection of point-like sources with unknown frequency spectra has been addressed.

First, a single-frequency case has been studied and limitations of this strategy has been highlighted by analytical and numerical arguments.

Afterwards, the Multi-Frequency MUSIC-like (I-MUSIC) algorithm presented in [9] was extended to the 2D-case and its performance was proved superior to the single-frequency case. The I-MUSIC also proved to be superior to other migration-based and conventional MUSIC-like methods. In detail, the I-MUSIC has been compared to the WB-MUSIC and a migration algorithm (as given by the formula $\left.\sum_{i}^{N_{f}}\left|\left\langle\mathbf{A}_{k}\left(\omega_{i}\right), \mathbf{u}_{1}\left(\omega_{i}\right)\right\rangle\right|^{2}\right)$ the latter not reported for the sake of shortness. It is shown that I-MUSIC enables a clearer and higher dynamic reconstruction by retaining resolution due to the highest frequencies.

The large number of cases investigated enable a deep understanding of the algorithms under test. In particular, the effects of mismatch between the permittivity of the background and host medium appeared in terms of delocalization error in the radial direction; whereas in pres- 
ence of a lossy host medium, resolution slightly degrades, too. The presented I-MUSIC algorithm proved also more efficient in terms of sensors number with a better performance compared to other techniques.

\section{ACKNOWLEDGMENT}

This work was supported by Ministry of University and Research (MIUR) through the Futuro in Ricerca (FIRB) initiative under the project MICENEA (RBFR12A7CD) and the COST Action IC1102 VISTA.

\section{APPENDIX A.}

Assume that radiated field is collected continuously over $\Sigma$. The set of radiated fields is thus a subset of the functional space of squared integrable complex valued functions supported over the observation domain, i.e., $E(\cdot) \in L^{2}(\Sigma)$.

Hence, it is a Hilbert space equipped with the scalar product defined as

$$
\langle f, g\rangle=\int_{0}^{2 \pi} f(\theta) g^{*}(\theta) d \theta
$$

with $f(\cdot) g(\cdot) \in L^{2}(\Sigma)$.

Recall now the Graf's addition formula for Hankel functions, i.e.,

$$
H_{0}^{(2)}\left(k_{B G}\left|\underline{r}_{O}-\underline{r}\right|\right)=\sum_{n} H_{n}^{(2)}\left(k_{B G} r_{O}\right) J_{n}\left(k_{B G} r\right) \exp \left[j n\left(\theta_{O}-\theta\right)\right]
$$

with $\underline{r}_{O} \equiv\left(r_{O}, \theta_{O}\right), \underline{r} \equiv(r, \theta), r_{O}>r$ and $J_{n}(\cdot)$ being Bessel functions of first kind. From Equations (A1) and (A2) it is obtained that

$$
\left\|H_{0}^{(2)}\left(k_{B G}\left|\underline{r}_{O}-\underline{r}\right|\right)\right\|^{2}=2 \pi \sum_{n}\left|H_{n}^{(2)}\left(k_{B G} r_{O}\right)\right|^{2} J_{n}^{2}\left(k_{B G} r\right)
$$

Analogously,

$$
\begin{aligned}
& \left\langle H_{0}^{(2)}\left(k_{B G}\left|\underline{r}_{O}-\underline{r}_{k}\right|\right), H_{0}^{(2)}\left(k_{B G}\left|\underline{r}_{O}-\underline{r}_{s}\right|\right)\right\rangle \\
= & 2 \pi \sum_{n}\left|H_{n}^{(2)}\left(k_{B G} r_{O}\right)\right|^{2} J_{n}\left(k_{B G} r_{k}\right) J_{n}\left(k_{B G} r_{S}\right) \times \exp \left[j n\left(\theta_{S}-\theta_{k}\right)\right](\mathrm{A} 4)
\end{aligned}
$$

If the source and the trial positions are not too close to the observation circle, then only $2 N=\max \left\{2 k_{B G} r_{S}, 2 k_{B G} r_{k}\right\}+1$ terms are relevant in the previous series [15]. Moreover, in this case the amplitude of Hankel function can be considered constant with index $n$. This is due to the 
asymptotic behavior of Hankel functions when the argument is greater than the order. Hence, Equations (A3) and (A4) can be approximated as

$$
\left\|H_{0}^{(2)}\left(k_{B G}\left|\underline{r}_{O}-\underline{r}\right|\right)\right\|^{2} \simeq 2 \pi\left|H_{0}^{(2)}\left(k_{B G} r_{O}\right)\right|^{2} \sum_{-N}^{N} J_{n}^{2}\left(k_{B G} r\right)
$$

and

$$
\begin{aligned}
& \left\langle H_{0}^{(2)}\left(k_{B G}\left|\underline{r}_{O}-\underline{r}_{k}\right|\right), H_{0}^{(2)}\left(k_{B G}\left|\underline{r}_{O}-\underline{r}_{s}\right|\right)\right\rangle \\
\simeq & 2 \pi\left|H_{0}^{(2)}\left(k_{B G} r_{O}\right)\right|^{2} \sum_{-N}^{N} J_{n}\left(k_{B G} r_{k}\right) J_{n}\left(k_{B G} r_{S}\right) \exp \left[j n\left(\theta_{S}-\theta_{k}\right)\right](\mathrm{A} 6)
\end{aligned}
$$

Accordingly, it is found that

$$
\begin{aligned}
\cos \eta_{k S} & =\frac{\sum_{n} J_{n}\left(k_{B G} r_{k}\right) J_{n}\left(k_{B G} r_{S}\right) \times \exp \left[j n\left(\theta_{S}-\theta_{k}\right)\right]}{\sqrt{\sum_{n} J_{n}^{2}\left(k_{B G} r_{S}\right) \sum_{n} J_{n}^{2}\left(k_{B G} r_{k}\right)}} \\
& =J_{0}\left(k_{B G}\left|\underline{r}_{k}-\underline{r}_{S}\right|\right)
\end{aligned}
$$

the latter being obtained once the summation index is restored (as it leads to little modification of results) by applying once again the addition theorem.

\section{APPENDIX B.}

Be $k_{B G}$ and $\tilde{k}_{B G}$ the assumed and the actual wavenumbers. In this case, Equation (A7) modifies as

$$
\cos \eta_{k S} \simeq \frac{\sum_{n} J_{n}\left(k_{B G} r_{k}\right) J_{n}\left(\tilde{k}_{B G} r_{S}\right) \times \exp \left[j n\left(\theta_{S}-\theta_{k}\right)\right]}{\sqrt{\sum_{n} J_{n}^{2}\left(\tilde{k}_{B G} r_{S}\right) \sum_{n} J_{n}^{2}\left(k_{B G} r_{k}\right)}}
$$

and can be rewritten as

$$
\cos \eta_{k S} \simeq J_{0}\left(k_{B G}\left|\underline{r}_{k}-\frac{\tilde{k}_{B G}}{k_{B G}} \underline{r}_{S}\right|\right)
$$

\section{APPENDIX C.}

Consider now the case the radiated field is collected over a finite set of $N_{O}$ points. In this case, the radiated field is a summable finite sequence (i.e., $E(\cdot) \in l_{N_{O}}^{2}$ ) and the scalar product is defined as

$$
\langle f, g\rangle=\sum_{n=1}^{N_{O}} f_{n} g_{n}^{*}
$$


In this case, Equation (A4) becomes

$$
\begin{aligned}
& \left\langle H_{0}^{(2)}\left(k_{B G}\left|\underline{r}_{O}-\underline{r}_{k}\right|\right), H_{0}^{(2)}\left(k_{B G}\left|\underline{r}_{O}-\underline{r}_{S}\right|\right)\right\rangle \\
= & \sum_{n, m} H_{n}^{(2)}\left(k_{B G} r_{O}\right) H_{n-m N_{O}}^{(1)}\left(k_{B G} r_{O}\right) J_{n}\left(k_{B G} r_{k}\right) J_{n-m N_{O}}\left(k_{B G} r_{S}\right) \\
& \exp \left[j n\left(\theta_{S}-\theta_{k}\right)\right] \exp \left[-j m N_{O} \theta_{S}\right]
\end{aligned}
$$

where without losing in generality $N_{O}$ has been assumed to be an odd number.

Now, reasoning as done in Equation (A7) and properly applying the addition theorem for Bessel functions, Equation (C2) can be approximated as

$$
\begin{aligned}
& \left|H_{O}^{(2)}\left(k_{B G} r_{O}\right)\right|^{2} \sum_{m} j^{m N_{O}} J_{-m N_{O}}\left(k_{B G}\left|\underline{r}_{k}-\underline{r}_{S}\right|\right) \\
& \exp \left[-j m N_{O} \arg \left(\underline{r}_{k}-\underline{r}_{S}\right)\right]
\end{aligned}
$$

Note that the term $j^{m N_{O}}$ takes into account phase differences between $H_{n}^{(2)}(\cdot)$ and $H_{n-m N_{O}}^{(1)}(\cdot)$ in Equation $(\mathrm{C} 2)$ and the term $\arg \left(\underline{r}_{k}-\underline{r}_{s}\right)$ instead means the phase of the difference vector $\underline{r}_{k}-\underline{r}_{s}$. Therefore, it follows that

$$
\begin{aligned}
\cos \eta_{k S} \simeq & J_{0}\left(k_{B G}\left|\underline{r}_{k}-\underline{r}_{S}\right|\right)+\sum_{m \neq 0} j^{m N_{O}} J_{-m N_{O}}\left(k_{B G}\left|\underline{r}_{k}-\underline{r}_{S}\right|\right) \\
& \times \exp \left[-j m N_{O} \arg \left(\underline{r}_{k}-\underline{r}_{S}\right)\right]
\end{aligned}
$$

\section{REFERENCES}

1. Bertero, M., "Linear inverse and ill-posed problems," Advances in Electronics and Electron Physics, Vol. 75, 1120-1989, Ed., P. W. Hawkes, Academic, New York, 1989.

2. Devaney, A. J. and G. C. Sherman, "Nonuniqueness in inverse source and scattering problems," IEEE Trans. Antennas Propag., Vol. 30, 1034-1037, 1982.

3. Langenberg, K. J., "Applied inverse problems for acoustic, electromagnetic and elastic wave scattering," Basic Methods of Tomography and Inverse Problems, P. C. Sabatier, Ed., Adam Hilger, Bristol, MA, 1987.

4. Pierri, R., A. Liseno, F. Soldovieri, and R. Solimene, "In-depth resolution for a strip source in the Fresnel zone," J. Opt. Soc. Am. A, Vol. 18, 352-359, 2001. 
5. Marengo, E. A. and A. J. Devaney, "The inverse source problem of electromagnetics: Linear inversion formulation and minimum energy solution," IEEE Trans. Antennas Propag., Vol. 47, 4102, 1999.

6. Solimene, R., A. Buonanno, and R. Pierri, "Imaging small PEC spheres by a linear $\delta$ approach," IEEE Trans. Geosc. Rem. Sen., Vol. 46, 3010-3018, 2008.

7. Yavuz, M. E. and F. L. Teixeira, "Frequency dispersion compensation in time reversal techniques for UWB electromagnetic waves," IEEE Geosc. Rem. Sen. Lett., Vol. 2, 233-237, 2005.

8. Joh, Y.-D., Y. M. Kwon, J. Y. Huh, and W.-K. Park, "Structure analysis of single- and 2 multi-frequency subspace migrations in 3 inverse scattering problems," Progress In Electromagnetics Research, Vol. 136, 607-622, 2013.

9. Solimene, R., A. Dell'Aversano, and G. Leone, "Interferometric time reversal MUSIC for small scatterer localization," Progress In Electromagnetics Research, Vol. 131, 243-258, 2012.

10. Schmidt, R. O., "Multiple emitter location and signal parameter estimation," IEEE Trans. Antennas Propag., Vol. 34, 276-280, 1986.

11. Odendaal, J. W., E. Barnard, and C. W. I. Pistorious, "Twodimensional super-resolution radar imaging using the MUSIC algorithm," IEEE Trans. Antennas Propag., Vol. 42, 1386-1391, 1994.

12. Gruber, F. K., E. A. Marengo, and A. J. Devaney, "Time-reversal imaging with multiple signal classification considering multiple scattering between the targets," J. Acoust. Soc. Am., Vol. 115, 3042-3047, 2004.

13. Giannopoulos, A., GprMax2D/3D, Users Guide, http://www.xmarks.com/site/www.gprmax.org/, 2002.

14. Yavuz, M. E. and F. L. Teixeira, "On the sensitivity of timereversal imaging techniques to model perturbations," IEEE Trans. Antennas Propag., Vol. 56, 834-843, 2008.

15. Brancaccio, A., G. Leone, and R. Pierri, "Information content of born scattered fields: Results in the circular cylindrical case," $J$. Opt. Soc. Am. A, Vol. 15, 1909-1917, 1998. 